\title{
USO DO HORMÔNIO DE CRESCIMENTO PARA O TRATAMENTO DA SÍNDROME DE TURNER
}

\section{USE OF GROWTH HORMONE FOR THE TREATMENT OS TURNER'S SYNDROME}

\author{
Suemi Paula Takahashi ${ }^{1}$; Astrid Wiens ${ }^{2}$; Andréia Cristina Conegero Sanches ${ }^{3}$
}

1.Farmacêutica pós-graduanda do Curso de Especialização em Farmacologia e Farmácia de Dispensação II da Universidade Estadual do Oeste do Paraná UNIOESTE.

2.Doutoranda do Programa de Pós-Graduação em Ciências Farmacêuticas Universidade Federal do Paraná

3.Farmacêutica, docente do curso de Farmácia da Universidade Estadual do Oeste do Paraná - UNIOESTE.

\section{RESUMO:}

A Síndrome de Turner é uma anormalidade cromossômica que acomete cerca de 0,1\% dos recém-nascidos do sexo feminino, caracterizada principalmente por baixa estatura e disgenesia gonadal. $\mathrm{O}$ tratamento para essa síndrome é baseado no uso do hormônio do crescimento $(\mathrm{GH})$. $\mathrm{O}$ objetivo desse trabalho foi realizar uma revisão na literatura a sobre o uso do $\mathrm{GH}$ na Síndrome de Turner. A dose inicial de aproximadamente $0,4 \mathrm{mg} / \mathrm{kg} / \mathrm{dia}$ é administrada via subcutânea no período da noite, e pode variar conforme a idade da criança, sendo que a idade inicial da terapia com GH um fator importante na determinação da eficácia do tratamento. Algumas reações adversas podem ocorrer, como edema, artralgias, mialgias, lombalgias, hipertensão e rinite. Estrogênios podem ser administrados concomitantemente para diminuir a perda da massa óssea na fase adulta, surgindo melhor efeito quando administrados via transdérmica ou intramuscular. Efeitos benéficos, como distensibilidade da parede aórtica e manutenção da função cardíaca normal também podem ser observados em pacientes utilizando o $\mathrm{GH}$. Estudos adicionais ainda são necessários para algumas questões relativas à eficácia e segurança do $\mathrm{GH}$ a longo prazo.

PALAVRAS-CHAVE: Síndrome de Turner; hormônio do crescimento; eficácia; segurança

\section{ABSTRACT:}

Turner syndrome is a chromosomal abnormality that affects about $0.1 \%$ of newborn females, mainly characterized by short stature and gonadal digenesis. Treatment for this syndrome is based on the use of Growth Hormone $(\mathrm{GH})$. The aim of this study was 
to review the literature about the use of $\mathrm{GH}$ in Turner Syndrome. The initial dose of approximately $0.4 \mathrm{mg} / \mathrm{kg} /$ day is recommended to be administered subcutaneously at night, and may vary by child's age, and the starting age of GH therapy, an important factor in determining the effectiveness of treatment. Some adverse reactions can occur, such as edema, arthralgia, myalgia, back pain, hypertension and rhinitis. Estrogens can be administered concurrently to reduce the loss of bone mass in adults, emerging best effect when administered transdermally or intramuscularly. Beneficial effects, such as the aortic wall distensibility and maintenance of normal cardiac function can also be observed in patients using $\mathrm{GH}$. Additional studies are still needed for some questions of safety and efficacy of $\mathrm{GH}$ in the long term therapy.

KEYWORDS: Turner Syndrome; Growth Hormone; efficacy; safety.

\section{INTRODUÇÃO}

Em 1938, Henry Turner descreveu uma síndrome caracterizada por baixa estatura, infantilismo sexual, pescoço alado e cúbito valgo, a Síndrome de Turner, também conhecida como a "síndrome monossômica XO" (JONES, 1998). Ela pode ser definida como a combinação de características físicas de ausência parcial ou completa de um dos cromossomos X. É a anormalidade cromossômica sexual mais comum em mulheres, que afeta um número estimado em $3 \%$ de todas as fêmeas concebidas. Estima-se que mais de $99 \%$ dos fetos com o cariótipo $45 X$ são abortados espontaneamente, especialmente no primeiro trimestre, apresentando uma taxa de prevalência de dois em cada 2500 recém-nascidos vivos do sexo feminino. Pensa-se que apenas os fetos com menos manifestações de anormalidades cromossômicas graves devem sobreviver. As características mais comuns da Síndrome de Turner são a baixa estatura e a disgenesia gonadal associada com outras características dimórficas (BATCH, 2002).

O linfedema é um dos diagnósticos ponteiros, que caracteriza a Síndrome de Turner no neonato. $\mathrm{O}$ linfedema congênito apresenta-se com protuberâncias residuais no dorso dos pododáctilos. Esta característica acarreta problemas ao longo da vida para essas meninas e mulheres com Síndrome de Turner, e pode se agravar no momento que se inicia o tratamento com os hormônios de crescimento e estrogênio (BATCH, 2002; JONES, 1998). O edema em pacientes com Síndrome de Turner é usualmente devido à obstrução linfática. Essas meninas tem propensão a formar queloides (SAENGER, 2004).

Uma série de anormalidades esqueléticas podem ser encontradas na Síndrome de Turner. As características mais comuns incluem desproporção esquelética como: cúbito valgo, quarto metacarpo e/ou metatarsos curtos, e também exostose tibial medial. Além disso, crianças com Síndrome de Turner, tem um risco 
luxação congênita do quadril (BATCH, 2002).

SAENGER (2004) demonstrou que, pacientes com síndrome de Turner, têm uma tendência aumentada para algumas anormalidades cardiovasculares, como coarctação da aorta, valva aórtica bicúspide, e mal formações cardiovasculares. Elas também podem apresentar problemas renais; hipertensão; hipotireoidismo (causada por tireoidite crônica auto-imune); otite média devido à distorção do desenvolvimento da trompa de Eustáquio; estrabismo. Problemas relacionados com estas anomalias devem ser considerados durante exames periódicos de acompanhamento.

SANDEN, ELING e OTTEN (2003) escreveram uma revisão bibliográfica sobre a Síndrome de Turner no período de 1962 aestudos neuropsicológicos e motores nessa síndrome, e concluíram que o desempenho do cociente de inteligência (PIQ) é menor na síndrome de Turner. Além disso, o processo de visão espacial é o principal problema cognitivo, e existem alguns indícios de uma relação de estruturas anatômicas neurais, disfunção hormonal e genótipo.

A Síndrome de Turner está associada ao aumento da resistência à insulina e adiposidade, que mais tarde poderia estar associada com diabetes tipo 2na vida dessas meninas (KIESS, KRATZCH e PFAEFFLE, 2006).té março de 2003, no que diz respeito a A baixa estatura é a característica clínica mais comum na Síndrome de Turner (SAENGER, 2004). Grande parte da deficiência de altura é causada por erro genético, ou melhor, a haploinsuficiência de baixa estatura homeobox, contendo gene (SHOX) localizado dentro da região do cromossomo pseudoautossomal X (RAPPOLD et al. 2002 e ELLISON et al., 1997 apud DAVENPORT e KEIZER-SCHAMA, 2006). O gene SHOX pertence a uma família de reguladores transcricionais, genes homeobox que são importantes controladores de processos de desenvolvimento. O SHOX mRNA e proteínas são encontrados em todas as zonas de crescimento fetal e infância (MUNNS et al., 2004 apud DAVENPORT e KEIZER-SCHAMA, 2006).

Atualmente o tratamento que está sendo utilizado para essas meninas que apresentam deficiência da produção do Hormônio de Crescimento $(\mathrm{GH})$ endógeno é o próprio Hormônio de Crescimento sintético. Assim, o objetivo desse trabalho é realizar uma revisão bibliográfica sobre o uso de GHna Síndrome de Turner.

\section{METODOLOGIA}

Foram avaliados artigos e livros que relatassem o uso do $\mathrm{GH}$ em pacientes com Síndrome de Turner. Os artigos foram buscados nas bases de dados: Science direct, Pubmed, Scielo, utilizando as palavras chave: Síndrome de Turner; Somatostatina e GH. Foram selecionados artigos publicados entre 1997 e 2007 . Foram buscadas informações relativas à terapia, como doses, via de administração, associação com outros medicamentos e idade do início do tratamento, além de 
reações adversas oriundas do tratamento.

\section{RESULTADOS E DISCUSSÃO}

Os seres humanos não respondem ao $\mathrm{GH}$ de espécies não primatas. $\mathrm{O}$ GH para uso terapêutico era antigamente purificado a partir da hipófise de cadáveres humanos e, portanto, só estava disponível em quantidades muito limitadas e era associado ao risco de doença de Creutzfeldt-Jakob. A produção de GH humano pela tecnologia do DNA recombinante não apenas aumentou a disponibilidade do hormônio, como também eliminou o risco de transmissão de doença associada ao hormônio derivado da hipófise (GOODMAN \& GILMAN, 2006; TRITOS e MANTZOROS, 1998).

Nos últimos 50 anos, o GH humano tem sido utilizado para o tratamento de alterações de crescimento. Esse uso tem se expandido desde 1985, quando foi possível sua biossíntese assim o tratamento de crianças com baixa quantidade desse hormônio tem alcançado sucesso (BUTLER, 2007). Recentemente o FDA (Food and Drug Administration), aprovou o uso desse hormônio em adultos com deficiência de GH, HIV com a síndrome desenvolvida por esse motivo, além de meninas com Síndrome de Turner epitomizadas (TRITOS e MANTZOROS, 1998).

A dose aprovada pelo FDA (Food and Drug Administration) é de inicialmente 0,375 mg / kg semanais (0,054 mg / kg / dia = 0,162 Ul / kg / dia - 4,8 Ul / $\left.\mathrm{m}^{2} / \mathrm{dia}\right)$. Existe um logaritmo, dose por efeito do $\mathrm{GH}$. Assim, a magnitude que o efeito do $\mathrm{GH}$ diminui, torna-se maior a dose desse GH (DAVENPORT e KEIZER-SCHAMA, 2006).

Para reproduzir o padrão normal de secreção, geralmente é administrada a crianças que têm deficiência de $\mathrm{GH}$, uma dose de $40 \mu \mathrm{g} / \mathrm{kg} / \mathrm{dia}$, por via subcutânea, à noite; embora a meia-vida circulante do GH seja de apenas 20 minutos, sua meia-vida biológica situa-se na faixa de 9 a $17 \mathrm{~h}$, sendo suficiente a sua administração uma vez/dia (GOODMAN \& GILMAN, 2006). Para meninas com Síndrome de Turner que geralmente são tratadas entre 2 a 5 anos de idade são empregadas doses um pouco mais elevadas, equivalentes a 0,05 mg/kg/dia (SAENGER, 2004; GOODMAN \& GILMAN, 2006). Para o GH obter melhores efeitos no tratamento do crescimento, ele deve ser administrado ao deitar-se. Recentemente, modelos matemáticos tem demonstrado que o GH é mais eficaz quando administrado sete vezes por semana (SORIANO-GUILLEN et al., 2005).

A idade inicial de terapia com $\mathrm{GH}$ é um fator importante na determinação da eficácia do tratamento. Geralmente, o tratamento é iniciado em meados da infância ou tardiamente, quando a menina apresenta altura inferior ao normal. Sabe-se, que a falha no desenvolvimento do crescimento inicia-se no útero, e prossegue a um ritmo rápido no pós-natal (DAVENPORT e KEIZER-SCHAMA, 2006). Estudos preliminares da Toddler Turner Study apud DAVENPORT e KEIZER-SCHAMA (2006), demonstraram 
que a terapia de GH é efetiva quando iniciada aos nove meses de idade.

BROECK et al. (1995) analisaram garotas com Síndrome de Turner, numa faixa etária de 10 a 14 anos, que estavam iniciando o tratamento com o $\mathrm{GH}$, num período de dois anos. Eles observaram, que nessas meninas que iniciavam o tratamento com $\mathrm{GH}$ a longo prazo, ou seja, com idades mais avançadas (>10 anos), a altura final foi apenas de três cm a mais, sendo assim não obtiveram um resultado tão desejado.

SORIANO- GUILLEN et al. (2005), demonstraram a importância de dois fatores agirem conjuntamente na terapia com GH na Síndrome de Turner, que são o início e a duração da terapêutica.

$\mathrm{O} \mathrm{GH}$ quando administrado em doses superiores ao recomendado pelo FDA, em tratamento para síndrome de Turner, está associado a uma diminuição da glicose plasmática (DAVENPORT e KEIZER-SCHAMA, 2006). As reações adversas são frequentes, especialmente as periféricas, tais como edema, artralgias, mialgias, lombalgias, hipertensão e rinite. Tais efeitos parecem estar relacionados com a dose e desaparecem com a redução das doses administradas. Além disso, elas parecem ser mais comuns entre os pacientes adultos com deficiência de início de tratamento, em relação àqueles que foram tratados desde o início da infância com $\mathrm{GH}$. A intolerância à glicose também tem sido relatada e parece resultar da resistência à insulina. Isso acontece mais em indivíduos com doenças graves ou em pacientes idosos saudáveis, embora esses efeitos colaterais sejam raros em crianças. A pancreatite aguda foi observada em número pequeno em crianças. O excesso de $\mathrm{GH}$ em adultos pode levar ao gigantismo (HINTZ, 1992 apud TRITOS e MANTZOROS, 1998). Também foi relatada a ocorrência de leucemia em algumas crianças tratadas com GH (GOODMAN \& GILMAN, 2006).

ROSENFELD et al. (1998), concluíram em seus estudos que, a terapia com GH isoladamente, ou em combinação com a oxandrolona, é capaz de estimular a curto prazo, o crescimento da estatura em meninas com Síndrome de Turner com diagnóstico precoce e início de tratamento. A velocidade de crescimento em meninas com Síndrome de Turner aumenta com o uso do $\mathrm{GH}$ quando administrado em doses elevadas e, associado com oxandrolona (PRICE e ALBERTSSON-WIKLAND, 1993 apud TRITOS e MANTZOROS, 1998).

Segundo GRAVHOLT e NAERAA (1997), o aumento na altura pelo uso do GH, estava acompanhado por um aumento ainda maior no tamanho dos pés. Dessa forma, concluiu-se que o crescimento desproporcional dos pés observada durante a terapêutica com $\mathrm{GH}$, poderia ser parte do desenvolvimento natural da Síndrome de Turner, mas que pode ser influenciado por doses mais elevadas de $\mathrm{GH}$.

Meninas com Síndrome de Turner possuem uma densidade óssea normal, relatada durante a infância, mas quando adultas, se não tratadas devidamente, podem apresentar baixa massa óssea, a qual está associada a um aumento de fraturas, 
principalmente fraturas osteoporóticas. A terapia adequada seria o $\mathrm{GH}$ associado aos estrogênios, que pode normalizar a densidade óssea na Síndrome de Turner (SAS et al., 2001).

DAVENPORT (2006) observou em seus estudos, que meninas com Síndrome de Turner, devem iniciar logo a terapia com o GH, para alcançar a altura desejada. E deve-se induzir a puberdade com tratamento de estrogênios transdérmicos ou intramusculares (IM), pois as análises revelaram que pacientes tratados com estradiol percutâneo atingiram uma altura 2,1 centímetros além daquelas que utilizaram estradiol por via oral. $\mathrm{E}$ as meninas que foram tratadas com estradiol intramuscular (IM), atingiram maior altura do que aquelas que iniciaram tardiamente o tratamento. Estes resultados são consistentes, pois mulheres adultas tratadas com estrogênios orais diminuem os níveis séricos de IGF-I e suprimem o IGF independente dos efeitos metabólicos do $\mathrm{GH}$, enquanto estrogênios transdérmicos não.

BANNINK et al. (2006) demonstraram uma situação normal de saúde relacionado à qualidade de vida de mulheres jovens com Síndrome de Turner após o tratamento com GH a longo prazo, e a indução da puberdade em uma idade e tempo apropriado. A relativa alta pontuação em algumas das escalas relacionadas com a saúde da qualidade de vida (QVRS) pode ser explicada por efeitos do estrógeno, ou seja, uma possível resposta a turnos, indica que as mulheres com Síndrome de Turner poderiam ter uma ou outra referência interna. Além disso, a satisfação com a altura e com o desenvolvimento mamário teve uma significativa influência positiva sobre as várias escalas da QVRS, incluindo o funcionamento social e físico.

BERG et al. (2006) estudaram os efeitos do uso do GH em pacientes com Síndrome de Turner sobre a dimensão e distensibilidade da aorta. E concluíram que, essas pacientes tratadas com $\mathrm{GH}$, apresentaram aortas dilatadas e sinais de diminuição da distensibilidade da parede aórtica. O tratamento com $\mathrm{GH}$ está diretamente ou indiretamente ligado aos efeitos benéficos sobre a biofísica das propriedades da parede aórtica na Síndrome de Turner. A gravidade das anomalias parece estar relacionada com a dose do $\mathrm{GH}$, com um efeito benéfico de uma maior dose desse hormônio sobre as anormalidades.

MATURA et al. (2007) concluíram em seus estudos, que o tratamento com GH em meninas com Síndrome de Turner sobre a dimensão ventricular esquerda, não diferem significativamente entre os grupos tratados e os não tratados com $\mathrm{GH}$, ou seja, o tratamento farmacológico com $\mathrm{GH}$ tem pouco efeito sobre o tamanho do ventrículo esquerdo (VE) em pacientes com Síndrome de Turner. Então, essas meninas não são especialmente vulneráveis à indução de hipertrofia cardíaca. Em conclusão, o tratamento com GH aumenta a estatura das meninas com a síndrome, mas não afeta de forma desproporcionada as dimensões cardíacas.

SILVERMAN e FRIEDLANDER (1997) demonstraram em seu estudo, que o 
uso de GH faz bem ao coração de adultos e crianças. O GH e o IGF-I desempenham papel importante no desenvolvimento e na manutenção da função cardíaca normal. Portanto, o GH em moderação, é bom para o coração dos adultos. Ao outro extremo, se não tratada a acromegalia, este está associado a um número muito mais elevado de problemas cardiovasculares em adultos. Crianças com Síndrome de Turner tratadas com GH em curto prazo, não apresentam complicações cardíacas. Mas nesse estudo permanecem algumas questões: a hipertrofia ventricular pode desenvolver-se a longo prazo, num período de 10 a 15 anos? A hipertrofia que se faz desenvolver nas crianças que são dadas a longo prazo na terapêutica farmacológica, é prontamente reversível quando a terapia é parada, como é o caso de acromegalia?

Novos estudos mostram uma nova preparação de depósito, durável por semanas a meses, pois as atuais injeções são subcutâneas, diárias. Com a descoberta de liberação de $\mathrm{GH}$ em peptídeos curtos, esses secretagogos seriam superiores ao $\mathrm{GH}$, que tem uma meia-vida relativamente curta. Então, com o tempo de meia-vida prolongado de depósito de $\mathrm{GH}$, liberam esses peptídeos, que podem ter ação maior que o GH, e que serão capazes de induzir a acromegalia (BUTLER, 2007).

\section{CONCLUSÃO}

O uso de GH em meninas com Síndrome de Turner é eficaz no desenvolvimento do crescimento da altura das mesmas. A dosagem normal administrada é de $0,05 \mathrm{mg} / \mathrm{Kg} / \mathrm{dia}$. Estudos demonstram que, para se obter um melhor resultado, essas meninas com Síndrome de Turner devem iniciar a terapêutica na infância, aos nove meses de idade, com resultado mais satisfatório em relação à altura desejada. Outro fator importante que devem caminhar conjuntamente é o início e a duração da terapêutica com $\mathrm{GH}$.

A terapia com $\mathrm{GH}$ isoladamente ou em associação com oxandrolona a curto prazo, é benéfica para a estimulação de $\mathrm{GH}$, mas quando administrada doses elevadas de $\mathrm{GH}$ associado à oxandrolona, esses aceleram a velocidade de crescimento. Porém, doses elevadas de $\mathrm{GH}$ isoladamente, podem levar a uma diminuição dos níveis de glicose e também a um aumento do tamanho do pés.

As meninas com Síndrome de Turner, também apresentam uma densidade óssea normal durante a infância, mas quando adultas, podem apresentar baixa massa óssea, a qual está associada a um aumento de fratura. Por isso ao entrarem na puberdade faz-se necessário, tratá-las com GH associado aos estrogênios, que pode normalizar a densidade óssea nessas garotas. Além disso, o estrogênio administrado por via percutânea e IM, apresenta melhor desenvolvimento na altura dessas meninas. Com o auxílio do estrogênio, elas teriam um desenvolvimento mamário normal, as quais apresentariam um melhor desempenho físico e social. 
O GH utilizado em meninas com Síndrome de Turner apresenta bons resultados em relação a problemas cardiovasculares. Pode-se concluir que a terapia com GH não leva a hipertrofia cardiovascular e apresenta sinais de diminuição da distensibilidade aórtica, sendo assim benéfico em curto prazo. Não há conhecimento se a terapia com GH em longo prazo pode levar a complicações cardíacas. Ainda, estudos demonstram que uma nova preparação de GH durável por semanas a meses ajudaria na melhora da qualidade de vida das pacientes com Síndrome de Turner. Entretanto, faz-se necessária a realização de estudos adicionais para se responder questões relacionadas à segurança e eficácia.

\section{REFERÊNCIAS}

BANNINK, E. M. N. et al. Quality of life after growth hormone therapy and induced puberty in women with Turner syndrome. The Journal of pediatrics. 95:101, 2006.

BATCH, J. Turner syndrome in childhood and adolescence. Best Practice \& Research. $16(3):$ 465:482, 2002.

BERG, J. et al. Aortic distensibility and dimensions and the effects of growth hormone treatment in the Turner syndrome. The American Journal of Cardiology. 1644:1649, 2006.

BROECK, J. et al. Final height after long-term growth hormone treatment in Turner syndrome. The Journal of Pediatrics. 127(5): 729:735, 1995.

BUTLER, G. Indications for growth hormone therapy. Pediatrics and Child Health. 17 (9): 356:360, 2007.

DAVENPORT, M. L. Evidence for early initiation of growth hormone and transdermal estradiol therapies in girls with Turner syndrome. Growth Hormone \& IGF Research. 16:91:97, 2006.

DAVENPORT, M. L.; KEIZER-SCHAMA, S. M. P. F. M. Growth and growth hormone treatment in Turner syndrome. International Congress Series. 1298: 33:41, 2006.

GOODMAN, L. S., GILMAN, A. As bases farmacológicas da terapêutica. $11^{a}$ Ed. Rio de Janeiro: MacGraw-Hill, 1344:1352, 2006.

GRAVHOLT, C. H.; NAERAA, R. W. Reference values for body proportions and body 
composition in adult women with Ullrich - Turner syndrome. Am. J. Med. Genet. 72(4): 403: 408, 1997.

JONES, K. L. Padrões reconhecíveis de malformações congênitas. $5^{\text {a }}$ Ed. São Paulo: Manole Ltda, 81:82, 1998.

KIESS, W; KRATZCH, J.; PFAEFFLE, R. The growth hormone (GH) and insulin-like growth factor (IGF) system in girls and women with Turner syndrome. International Congress Series. 1298:63:70, 2006.

MATURA, L. A. et al. Growth hormone treatment and left ventricular dimensions in Turner syndrome. The Journal of pediatrics. 587:591, 2007.

ROSENFELD, R. et al. Growth hormone therapy of Turner's syndrome: Beneficial effect on adult height. The Journal of Pediatrics. 132(2): 319-324, 1998.

SAENGER, P. Transition in Turner's Syndrome. Growth Hormone \& IGF Research. 14: 72:76, 2004.

SANDEN, M., ELING, P., e OTTEN, B. A review of neuropsychological and motor studies in Turner Syndrome. Neuroscience and Biobehavioral Reviews. 27: 329:338, 2003.

SAS, T. C. et al. Bone mineral density assessed by phalangeal radiographic absorptiometry before and during long-term growth hormone treatment in girls with Turner's syndrome participating in a randomized dose-response study. Pediatric Research. 50(3):417:422, 2001.

SILVERMAN, B. L.; FRIEDLANDER, J. R. Is growth hormone good for the heart? The Journal of pediatrics. 131(1): 70:74, 1997.

SORIANO-GUILLEN, L. et al. Adult height and pubertal growth in Turner syndrome after treatment with recombinant growth hormone. J. Clin. Endocrinol. Metab.: 90(9): 5197: 5204, 2005.

TRITOS, N. A.; MANTZOROS, C. S. Recombinant human growth hormone: old and novel uses. The American Journal of Medicine. 105: 44:57, 1998. 